\title{
PAHs in Seawater of the Semi-Closed Areas along the Alexandria Coast of Egyptian Mediterranean Sea
}

\author{
Mohamed A. Shreadah ${ }^{1^{*}}$, Mohamed I. Abdel Moneim ${ }^{1}$, Tarek O. Said ${ }^{2}$, Eiman M. I. Fathallah ${ }^{3}$, \\ Mohamed E. Mahmoud ${ }^{3}$ \\ ${ }^{1}$ National Institute of Oceanography and Fisheries, Alexandria, Egypt; ${ }^{2}$ Chemistry Department, Faculty of Science, King Khalid \\ University, Abha, KSA; ${ }^{3}$ Chemistry Department, Faculty of Science, Alexandria University, Alexandria, Egypt. \\ Email: ${ }^{*}$ m_shreadah@yahoo.com, ${ }^{*}$ niof@hotmail.com
}

Received August $27^{\text {th }}, 2013$; revised September $29^{\text {th }}, 2013$; accepted October $26^{\text {th }}, 2013$

Copyright (C) 2013 Mohamed A. Shreadah et al. This is an open access article distributed under the Creative Commons Attribution License, which permits unrestricted use, distribution, and reproduction in any medium, provided the original work is properly cited.

\begin{abstract}
The distributions of 15 out of 16 polycyclic aromatic hydrocarbons (PAHs) were determined in surface and bottom water of Alexandria coasts (Egyptian Mediterranean Sea). Total average PAH concentrations were ranged from 9.89 to $677.25 \mathrm{ng} / \mathrm{l}$, and from 2.21 to $345.29 \mathrm{ng} / \mathrm{l}$ in surface and bottom water, respectively. PAH concentrations were at relatively low levels compared to those reported for other estuaries and marine systems around the world. The $\Sigma$ COMB and $\Sigma T F-P A H s$ suggest the origin of hydrocarbons. The higher concentrations of total pyrolytic hydrocarbons ( $\Sigma \mathrm{COMB})$ than that of total fossil hydrocarbons ( $\mathrm{TFPAH})$ declared that atmospheric fall-out from traffic exhausts was the significant source of PAHs to marine environment of the semi-closed areas of Alexandria. Interferences of rather petrogenic and pyrolytic PAH contaminations were noticed in the harbours, especially marine area due to petroleum products deliveries and fuel combustion emissions from the ships awaiting along side the quays.
\end{abstract}

Keywords: Surface Seawater; Bottom Seawater; PAHs; Alexandria; Egypt; GC-MS

\section{Introduction}

Water pollution is a serious problem in the global context. Marine environments are considered to be the ultimate pool of many organic pollutants [1-3] introduced directly or indirectly through anthropogenic and/or natural sources. The study of trace organic contaminants in coastal marine environment is of great importance, since these areas are biologically productive and receive considerable pollutant inputs from land-based sources via point and nonpoint sources [4-7]. The quality of the aquatic ecosystem is of great interest to the entire world. Scientific interest in the quality of marine ecosystems is quite recent and has especially increased in the past 15 years in relation to application of the European Union (EU)'s Water Framework Directive (WFD) [8]. One of the main objectives of the WFD is achievement and preservation of "Good Chemical Status" of surface waters of the EU member states. Of all marine pollutants, hydrocarbons have received the greatest attention due primarily to the highly lethal effects of most hydrocarbons on marine organisms, espe-

${ }^{*}$ Corresponding author. cially aromatics class. The occurrence of PAHs in marine environment has attracted the attention of the scientific community, as these compounds are frequently detected in seawater and sediments at increasing levels and have adverse health effects on marine organisms and humans. Several PAHs are potential human carcinogens and are included in the priority list of the European Union Water Framework Directive (2000/60/EC). Sixteen PAHs, identified by the US Environmental Protection Agency (EPA) as priority pollutants [9-14], are a class of organic pollutants which have an important impact on marine ecosystems because they include the largest known class of chemical carcinogens and mutagens [15-17]. This study aims to assess the distributions and characteristics of selected parent PAHs in aqueous phase along Alexandria coast and the correlation of the physicochemical parameters on their distribution. Conversely, in the Eastern Mediterranean few data have been published on the presence of PAHs in coastal water close to point sources (municipal and river discharges, etc.). In addition, hydrocarbon budgets are available for the Western part of Mediterranean Sea [18-20], but there is a tremendous lack of informa- 
tion regarding the Southern Mediterranean [21-28]. This is the first systematic study to be undertaken along the semi-closed areas of Alexandria (Egyptian Mediterranean Sea coast) as a comparative study between four sectors of different identity. The available data of hydrocarbon levels in the Egyptian marine environment are scarce in the last ten years [23-30]. Along the Mediterranean coast of Alexandria city, there are many areas with high activities of shipping and pleasure boating activities, incorporating numerous Harbours and marina [28-39].

\section{Materials and Methods}

\subsection{Sampling Plan}

Water samples were collected from 49 sampling stations; (49 surface water $\& 43$ bottom water samples), along the semi-closed areas of Alexandria coasts, Egypt as shown in (Figure 1).

Surface and bottom water samples were collected from 49 stations distributed along the semi-closed areas of Alexandria (Egyptian Mediterranean Sea coast), namely Abu-Qir Bay (AQ), Eastern Harbour (EH), Western Harbour (WH), and El-Mex Bay (Max) during 2010 on local motorboat using Niskin bottle. The location of each sampling station was determined using a GPS (Trimble Navigation, Japan) to pinpoint sampling locations. Sampling sites were selected taking into consideration the expected polluted areas due to industrial and human impacts. Temperature and $\mathrm{pH}$ values were obtained at each site by deploying a multi-variable sensor. A set of water samples were fixed to determine dissolved oxygen (DO), also oxidizable organic matter (OOM) was determined in all samples. Samples were subsequently kept in are frigerator at $-4^{\circ} \mathrm{C}$ prior to analysis in the laboratory.

\subsection{Extraction}

\subsubsection{Physicochemical Characteristics of Water Samples}

Salinity (\%) was measured in situ using a Salinometer equipment (Thermo Electron Corporation 088749, USA). $\mathrm{pH}$ was measured in the field by the use of a $\mathrm{pH}$ meter equipment (JENWAY 3505, UK), DO was fixed immediately and determined according to the classical Winkler method (Strickland and Parsons) [40]. OOM was done as described by FAO [41].

\subsubsection{Determination of Petroleum Hydrocarbons in Water}

One liter of seawater was poured into glass separatory funnel, extracted twice with $2 \times 40 \mathrm{ml}$ of methylene chloride. The extract was stored in dark at low temperature $\left(\sim 5^{\circ} \mathrm{C}\right)$. Before analysis, the stored extracts were evaporated to dryness in a rotary evaporator at $30^{\circ} \mathrm{C}$ under reduced pressure. The residue was re-dissolved in $1 \mathrm{ml}$ of n-hexane before fractionation with column chromatography [42]. The extracted volumes were passed through the silica column prepared by slurry packing $20 \mathrm{mls}(10$ g) of silica (activated at $185^{\circ} \mathrm{C}$ overnight and deactivated with $12.5 \%$ water afterwards), followed by $10 \mathrm{mls}(10 \mathrm{~g})$ of alumina (90 neutral, 70 - 230 mesh previously activated at $185^{\circ} \mathrm{C}$ overnight and deactivated with $12.5 \%$ water afterwards) and finally $1 \mathrm{~g}$ of anhydrous sodium sulphate. Elution was performed using $40 \mathrm{mls}$ of hexane (aliphatic fractions), then $40 \mathrm{mls}$ of hexane/dichloromethane (90:10) followed by $20 \mathrm{mls}$ of hexane/dichloromethane (50:50) (which combined contain PAHs). Finally, the eluted samples were concentrated under a gentle stream of $\mathrm{N}_{2}$ to about $0.2 \mathrm{ml}$. Activated copper was added for desulfurization [43].

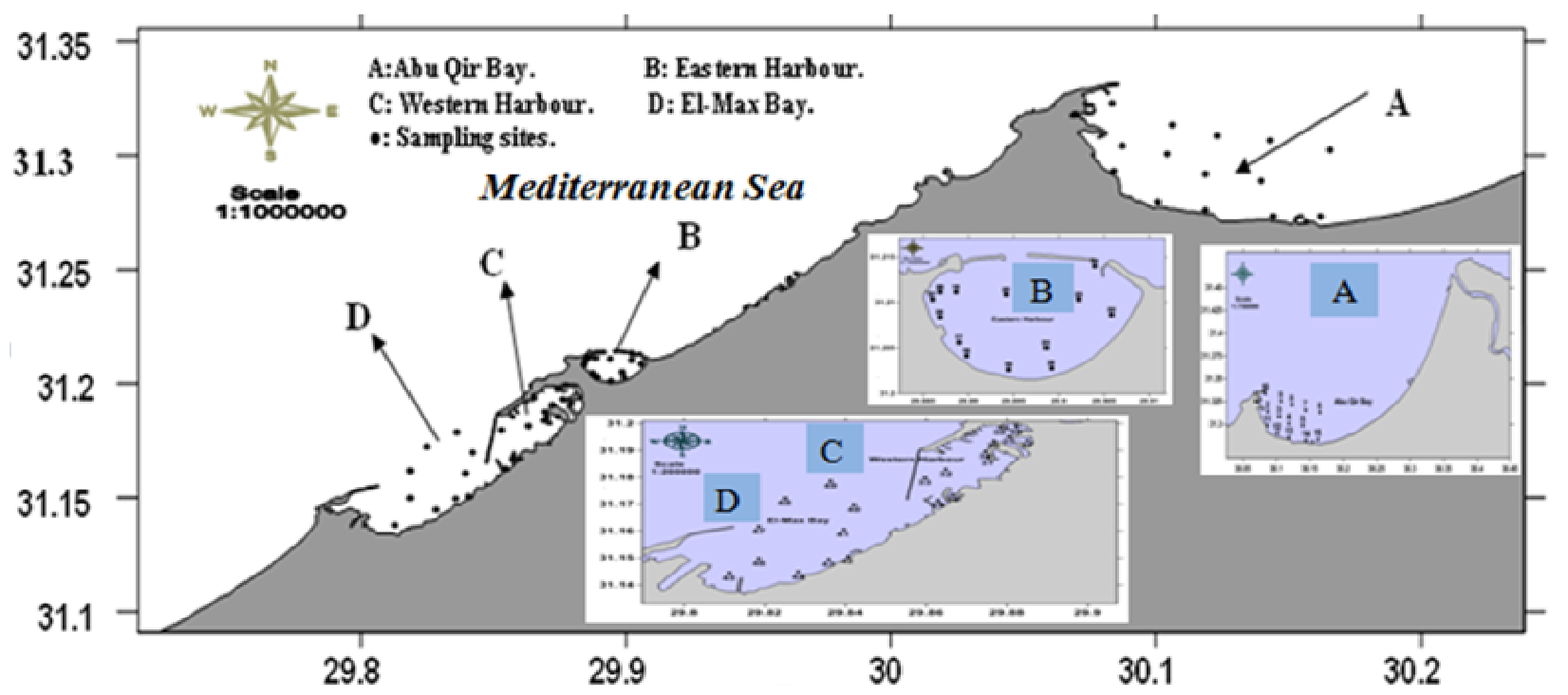

Figure 1. Study area along Alexandria Coasts of the Egyptian Mediterranean Sea. 


\section{1) Blank preparation}

According to Parson et al., [42] eighty mls of methylene chloride were poured into a rotary evaporator flask and evaporated to dryness at $30^{\circ} \mathrm{C}$. The residue was dissolved in $1 \mathrm{ml}$ of $\mathrm{n}$-hexane before fractionation.

2) Identification of PAHs using Gas chromatography-Mass spectrometry (GC-MS)

Blanks of 1000 fold concentration were analyzed by Gas Chromatograph-Mass Spectrometer; Trace-Ultra coupled to DSQ-II MS (Thermo Electron SPA, UK) equipped with auto injector. The mass spectrometer was operated in full scan mode (50 - 650) Daltons per second. The ionization source was supplied with voltage at $70 \mathrm{eV}$. Three $\mu$ l volume of each sample was injected in the splitless mode. The 15 out of 16 EPA-PAH compounds were naphthalene (Naph, m/z 128), acenaphthylene (Acthy, $\mathrm{m} / \mathrm{z}$ 152), acenaphthene (Ace, $\mathrm{m} / \mathrm{z} 154$ ), phenanthrene (Phe, $\mathrm{m} / \mathrm{z}$ 178), anthracene (Ant, $\mathrm{m} / \mathrm{z}$ 178), fluoranthene (Flu, m/z 202), pyrene (Pyr, m/z 202), benzo(a)anthracene $(\mathrm{BaA}, \mathrm{m} / \mathrm{z} 228)$, chrysene $(\mathrm{Chr}, \mathrm{m} / \mathrm{z} 228)$, benzo (b)fluoranthene $(\mathrm{BbF}, \mathrm{m} / \mathrm{z}$ 252), benzo(k)fluoranthene (BkF, m/z 252), benzo(a)pyrene (BaP, m/z 252), benzo (ghi)perylene (BghiP, m/z 276), indeno (1,2,3-cd)pyrene (InP, m/z 276), and dibenz(a,h) anthracene (DBA, m/z 278). GC/MS is equipped with splitless injector and a fused silica capillary column; Thermo TR-35 MS (30m, $0.25 \mathrm{~mm}, 0.25 \mu \mathrm{m})$ with $35 \%$ phenyl polysilphenylenesiloxane. Helium was used as carrier gas at $1.5 \mathrm{ml} \cdot \mathrm{min}^{-1}$. The oven temperature program was $60^{\circ} \mathrm{C}(1 \mathrm{~min})$ ramped at $8^{\circ} \mathrm{C} / \mathrm{min}$ to $100^{\circ} \mathrm{C}$, hold for 1 minute then ramped at $5^{\circ} \mathrm{C} / \mathrm{min}$ to $300^{\circ} \mathrm{C}$ maintaining about $20^{\circ} \mathrm{C}$. The injection and the transfer line temperature were maintained at $280^{\circ} \mathrm{C}$ and $300^{\circ} \mathrm{C}$, respectively.

The response factor of individual $\mathrm{PAH}$ compounds to the internal standard was measured and calculated at least three times at the beginning, in the middle and at the end for each batch of GC injections (15 samples). Identification and quantification of 15 out of 16 EPAPAH compounds were based on matching their retention times with a mixture of PAH standard.

\section{3) Analytical quality control}

To control the analytical reliability and assure recovery efficiency and accuracy of the results, 6 analyses were conducted on PAH compound reference materials, HS-5 (sediments) provided by NRC-IMB of Canada and SRM-2974 (Freeze-dried mussels tissue) (Mytilusedulis) provided by NIST of USA as well as artificial seawater samples of known PAH levels spiked with a mixture consisting of $2 \mu \mathrm{g}$ each of PAHs were analyzed as above to validate the analytical method used in this study. The recovery efficiency ranged from $92 \%$ to $111 \%$ for HS-5, $88 \%$ to $96 \%$ for SRM-2974 and $93 \%$ to $105 \%$ for the spiked samples. The mean recovery for PAHs were as followed: Naph 90.1\%, Acthy 92.3\%, Ace 105.2\%, Flu
95.4\%, Pyr 92.5\%, BaA 89.7\%, Chr 107.1\%, BaP 94.6\%, BbF 90.5\%, DBA 101.8\%, BghiP 93.7\% and InP 97.5\%.

\section{Results and Discussion}

\subsection{PAHs in Water Samples}

Tables 1(a)-(d) showed the total concentrations of the PAHs in water. The concentrations below their limits of detection were given a value of zero for the calculation. ( $\mathrm{PAH}$ ) concentrations in water varied significantly among the studied locations. The values ranged from 2.2 to $677.25 \mathrm{ng} / \mathrm{l}$ with an average value of $192.29 \mathrm{ng} / \mathrm{l}$. The highest concentration of total PAHs was measured in water collected from St. 6S (AQ), while lower concentrations were detected in sample of St. 2B (AQ). In this study, the order of decreasing PAHs concentrations for surface and bottom waters is illustrated in Figure 2. The average concentration of PAHs in the present study was moderate in comparison to those in most areas of urbanized estuaries in the other countries represented in Table 2.

\subsection{PAHs and OOM}

No correlation between hydrocarbons and organic matter in water column where; $(\mathrm{R}=-0.2368, \mathrm{n}=92)$. This may attribute to the weak ability of hydrocarbons to adsorb onto organic matter in water phase at low concentrations of PAHs (Figure 3).

\subsection{Comparison of Different PAHs Fractions in Water Samples of the Four Areas}

Figures 4 and $\mathbf{5}$ showed the percentages of the average combustion, carcinogenic and fossil PAHs of the four sectors to each other. There is no significant difference in percentages of combustion PAHs in AQ, EH and $\mathrm{WH}$ in surface and bottom water and they are larger than that calculated for El-Max Bay. In contrast, Max possesses the highest percentage of fossil PAHs may be interpreted that the anthropogenic sources are loaded mostly with LMW PAHs (molecular weight $<178$ ) which have ability to dissolve in water than HMW PAHs (molecular weight $>178$ ), so the percent of saturation of fossil PAHs becomes $45 \%$ or more greater than that belong to combustion PAHs $<30 \%$. This may give indication that the origin of PAHs in AQ, EH, and $\mathrm{WH}$ are generally pyrogenic; while it is petrogenic in max.

\subsection{Physicochemical Parameters in Water Samples}

Tables 3 and $\mathbf{4}$ showed some physic-chemical parameters; Salinity \%o, pH, OOM (mg/l), DO ( $\left.\mathrm{mgO}_{2} / \mathrm{l}\right), \Sigma \mathrm{PAHs}(\mathrm{ng} / \mathrm{l})$, $\Sigma$ COMP (ng/l), $\Sigma$ CARC (ng/l), and $\Sigma$ TFPAH (ng/l) in 
Table 1. (a) Concentrations (ng/l) of PAHs in water samples collected from Abou Qir (AQ) during 2010; (b) Concentrations (ng/l) of PAHs in water samples collected from Easter Harbor (EH) during 2010; (c) Concentrations (ng/l) of PAHs in water samples collected from Western Harbor (WH) during 2010; (d) Concentrations (ng/l) of PAHs in water samples collected from El-Max (Max) during 2010.

(a)

\begin{tabular}{|c|c|c|c|c|c|c|c|c|c|c|c|c|c|c|c|}
\hline $\begin{array}{c}\text { Parameter } \\
\text { Site }\end{array}$ & Naph & Acthy & Ace & Phe & Ant & Flu & Pyr & $\mathbf{B a A}$ & Chr & BbF & BkF & BaP & InP & B(ghi)P & DBA \\
\hline $1 \mathrm{~S}$ & 10.56 & 9.6 & 4.8 & 9.6 & 7.2 & 23.76 & 2.4 & 17.6 & 67.2 & 99.92 & 52.8 & 21.6 & 21.08 & 10.32 & 6.88 \\
\hline $2 \mathrm{~S}$ & 17.68 & ND & 6.73 & 2.6 & 10.4 & 62.4 & 6.98 & 26 & 28.6 & 83.2 & 13 & 39.78 & 109.08 & 101.14 & 67.43 \\
\hline $2 \mathrm{~B}$ & ND & ND & $<\mathrm{DL}$ & $<\mathrm{DL}$ & $<\mathrm{DL}$ & $<\mathrm{DL}$ & ND & ND & $<\mathrm{DL}$ & $<\mathrm{DL}$ & $<\mathrm{DL}$ & $<\mathrm{DL}$ & ND & ND & $<\mathrm{DL}$ \\
\hline $3 \mathrm{~S}$ & 7.56 & $<\mathrm{DL}$ & 3.6 & 9.89 & 3.6 & 45.97 & 1.8 & 13.8 & 7.2 & 52.2 & 14.4 & 97.2 & 17.83 & 78.48 & 52.32 \\
\hline $3 B$ & ND & ND & ND & $<\mathrm{DL}$ & $<\mathrm{DL}$ & $<\mathrm{DL}$ & ND & 7.93 & ND & 5.77 & 12.8 & 3.98 & 17.95 & 78.2 & 52.13 \\
\hline $4 \mathrm{~S}$ & 8.36 & ND & 3.8 & 18.68 & 5.3 & 41.8 & $<\mathrm{DL}$ & 13.3 & 9.5 & 24.7 & 17.1 & 76 & 13.25 & 156.37 & 104.25 \\
\hline $4 B$ & ND & ND & 6.4 & $<\mathrm{DL}$ & 10.98 & 3.7 & ND & 5.3 & 3.15 & 18.2 & 11.09 & 3.92 & 26. & 57.2 & 38.13 \\
\hline $5 \mathrm{~S}$ & 19.32 & $<\mathrm{DL}$ & 5.67 & 4.6 & 11.5 & 46.59 & 2.3 & 7.93 & 16.1 & & 85.1 & 27.6 & 7. & 38.64 & 25.76 \\
\hline $5 \mathrm{~B}$ & 6.72 & ND & ND & 8.71 & $<\mathrm{DL}$ & 2.4 & $<\mathrm{DL}$ & 8 & 12.88 & & 24 & 7.2 & 27. & 49.44 & 32.96 \\
\hline $6 \mathrm{~S}$ & 6.24 & $<\mathrm{DL}$ & 5.7 & 3.5 & 5.2 & 11.45 & 2.6 & 11.27 & 7.8 & 13.28 & 109.2 & 49.4 & 10.24 & 28.5 & 19 \\
\hline $6 \mathrm{~B}$ & ND & ND & ND & $<\mathrm{DL}$ & 2.3 & 16.96 & $<\mathrm{DL}$ & 5.37 & 4.6 & 10.26 & 20.7 & 4.83 & 7.75 & 24.61 & 16.41 \\
\hline $7 \mathrm{~S}$ & 11 & $<\mathrm{DL}$ & 10 & 2.5 & 12.5 & 50.98 & 9.84 & 12.5 & 17.5 & 11 & 192.5 & 112.5 & 18 & 49 & 32.67 \\
\hline $7 \mathrm{~B}$ & ND & ND & $<\mathrm{DL}$ & 2.6 & 4.7 & NI & ND & ND & ND & 13. & 15.23 & 19.45 & 3. & 15.08 & 10.05 \\
\hline $8 \mathrm{~S}$ & 17.48 & $<\mathrm{DL}$ & $<\mathrm{DL}$ & $<\mathrm{DL}$ & 3 & & 1.9 & 46.87 & 22.8 & 10 & 32 & 47 & 52. & 47. & 31.54 \\
\hline $8 \mathrm{~B}$ & ND & ND & $<\mathrm{DL}$ & $<\mathrm{DL}$ & 1.8 & 3.65 & $<\mathrm{DL}$ & 9.6 & 1.8 & 7.2 & 13.76 & 10.8 & 8.24 & 51.12 & 34.0 \\
\hline $9 \mathrm{~S}$ & 7.22 & ND & 1.9 & 13.6 & 7.4 & 7.6 & $<\mathrm{DL}$ & 9.5 & 3.8 & 11.4 & 43.7 & 70.3 & 19.95 & 48.83 & 32.55 \\
\hline $9 \mathrm{~B}$ & & 1.6 & $<\mathrm{DL}$ & 8.3 & 3.2 & 6.4 & 1.6 & 31.47 & 3.2 & & 6.4 & 16 & 17. & 3.26 & 2.18 \\
\hline $10 \mathrm{~S}$ & 10.08 & 1. & 3.6 & 5.4 & 4.3 & 7. & 1.8 & 13.87 & 12.6 & 73. & 12.6 & 34.2 & 27.79 & 124.02 & 82.68 \\
\hline $10 \mathrm{~B}$ & & $\mathrm{~N}$ & & $<\mathrm{DL}$ & 1. & 1. & $<\mathrm{DL}$ & & ND & 3. & 3.8 & 5.7 & 10.4 & 23.56 & 15.71 \\
\hline $11 \mathrm{~S}$ & 19.72 & 1. & 12.79 & $<\mathrm{DL}$ & 3.4 & 6.8 & $<\mathrm{DL}$ & 4.5 & 5.1 & 61.2 & 6.8 & 39.1 & 16. & 45.9 & 30.6 \\
\hline $12 \mathrm{~S}$ & & 2.4 & 2.4 & 7.2 & 4.8 & 21.6 & 2.4 & 37.6 & 7.2 & 9.6 & 12.56 & 43.2 & 26.32 & 157.44 & 104.96 \\
\hline $13 \mathrm{~S}$ & 9.86 & 23.65 & 1.7 & 5.1 & 3.4 & 15.3 & 1.7 & 12.47 & 11.9 & 35.7 & 18.7 & 27.2 & 55.46 & 17.51 & 11.67 \\
\hline $14 \mathrm{~S}$ & 10.92 & 7.9 & 13.45 & 23.01 & 10.98 & 28.6 & 2.6 & 30.33 & 13 & 36. & 12.98 & 23.4 & 9.59 & 17.32 & 11.54 \\
\hline $15 \mathrm{~S}$ & 7.6 & 15.65 & 10.76 & 3.8 & 10.45 & 22.8 & $<\mathrm{DL}$ & 16.47 & 3.8 & 13.3 & 7.6 & 41.8 & 17.73 & 26.98 & 17.99 \\
\hline
\end{tabular}

Naph: Naphthalene, Acthy: Acenaphthylene, Ace: Acenaphthene, Phe: Phenanthrene, An: Anthracene, Flu: Fluoranthene, Py: Pyrene, BaA: Benzo(a)anthracene, Chr: Chrysene, BkF: Benzo(k)flouranthene, BaP: Benzo(a)pyrene, BbF: Benzo(b)fluoranthene, InP: Indenopyrene, B(ghi)P: Benzo(g,h,i)perylene, DBA: Dibenz(a,h)anthracene, ND: Not detected, <DL: Lower detection limit, S: Surface, and B: Bottom.

(b)

\begin{tabular}{|c|c|c|c|c|c|c|c|c|c|c|c|c|c|c|c|}
\hline $\begin{array}{c}\text { Parameter } \\
\text { Site }\end{array}$ & Naph & Acthy & Ace & Phe & Ant & Flu & Pyr & $\mathbf{B a A}$ & Chr & $\mathbf{B b F}$ & BkF & $\mathrm{BaP}$ & InP & B(ghi)P & DBA \\
\hline $15 \mathrm{~S}$ & 7.6 & 15.65 & 10.76 & 3.8 & 10.45 & 22.8 & $<\mathrm{DL}$ & 16.47 & 3.8 & 13.3 & 7.6 & 41.8 & 17.73 & 26.98 & 17.99 \\
\hline $16 \mathrm{~S}$ & 6.05 & 2.5 & 8.2 & 14.23 & 3.7 & 2.5 & 9.8 & 16.67 & 19.8 & 35 & 13.89 & 11.87 & 10.48 & 78.5 & 52.33 \\
\hline $16 \mathrm{~B}$ & 25.48 & 10.4 & 23.76 & $<\mathrm{DL}$ & 5.2 & 15.82 & 47.18 & 28.6 & 5.2 & 12.65 & 15.87 & 10.18 & 7.61 & 13.78 & 9.19 \\
\hline $17 \mathrm{~S}$ & 19.36 & 35.2 & 12.26 & $<\mathrm{DL}$ & 2.2 & 7.56 & $<\mathrm{DL}$ & 16.13 & 6.6 & 16.92 & 11.29 & 8.39 & 40.38 & 75.9 & 50.6 \\
\hline $17 \mathrm{~B}$ & 15.2 & 3.8 & 12.56 & 19.76 & 9.4 & 13.7 & 1.9 & 14.57 & 12.5 & 13.15 & 16.4 & 1.9 & 8.54 & 26.22 & 17.48 \\
\hline $18 \mathrm{~S}$ & 11.02 & 30.6 & 10.8 & 6.7 & 3.6 & $<\mathrm{DL}$ & $<\mathrm{DL}$ & 22.2 & 12.76 & 10.8 & 18 & 7.2 & 20.99 & 13.68 & 9.12 \\
\hline $18 \mathrm{~B}$ & 15.04 & 12.8 & 4.87 & 1.6 & $<\mathrm{DL}$ & 4.87 & $<\mathrm{DL}$ & 22.4 & 13.87 & 6.4 & 5.21 & 3.2 & 5.33 & 15.04 & 10.03 \\
\hline $19 \mathrm{~S}$ & 29.4 & 30 & 13.45 & $<\mathrm{DL}$ & 12.5 & $<\mathrm{DL}$ & 34.21 & 18 & 17.13 & 5.98 & 23.98 & 2.94 & 26.75 & 41.4 & 27.6 \\
\hline $19 \mathrm{~B}$ & 13.64 & 15.4 & 2.2 & 13.53 & 8.5 & 6.6 & 12.87 & 8.8 & 15.4 & 21.87 & $<\mathrm{DL}$ & 2.2 & 6.96 & 31.9 & 21.27 \\
\hline $20 \mathrm{~S}$ & 73.44 & 4.8 & 12.34 & 5.4 & 4.8 & 13.87 & 3.7 & 8 & 7.2 & 13.29 & 21.26 & 3.84 & 20.5 & 103.92 & 69.28 \\
\hline $20 \mathrm{~B}$ & 11.84 & 13.87 & 1.6 & $<\mathrm{DL}$ & 13.72 & 3.2 & $<\mathrm{DL}$ & 8 & 15.43 & 1.6 & 10.65 & 13.98 & 5.11 & 11.36 & 7.57 \\
\hline $21 \mathrm{~S}$ & 6.56 & 35.7 & 6.8 & 1.7 & 21.5 & 1.7 & $<\mathrm{DL}$ & 14.17 & 15.3 & 13.6 & 5.93 & 9.63 & 20.82 & 56.27 & 37.51 \\
\hline 21B & 13.5 & 19.5 & 3 & $<\mathrm{DL}$ & 3.8 & 12.7 & 10.98 & 14 & 9 & 3.6 & 2.7 & 5.6 & 2.62 & 20.1 & 13.4 \\
\hline $22 \mathrm{~S}$ & 8.64 & 9.6 & 17.6 & 8.5 & 3.2 & $<\mathrm{DL}$ & $<\mathrm{DL}$ & 11.2 & 8 & 5.11 & 12.6 & 3.29 & 10.1 & 39.84 & 26.56 \\
\hline $22 \mathrm{~B}$ & 14.06 & 5.7 & 3.8 & $<\mathrm{DL}$ & 1.9 & 3.5 & 1.9 & 19.63 & 7.6 & 3.8 & 3.8 & 6.56 & 10.56 & 14.06 & 9.37 \\
\hline $23 \mathrm{~S}$ & 10 & 2.5 & 13.23 & $<\mathrm{DL}$ & 3.65 & 3.98 & $<\mathrm{DL}$ & 15.83 & ND & 4.76 & $<\mathrm{DL}$ & 2.5 & 3.35 & 19.25 & 12.83 \\
\hline $23 \mathrm{~B}$ & 34.08 & 2.4 & 6.47 & ND & 5.47 & $<\mathrm{DL}$ & $<\mathrm{DL}$ & 16 & 7.2 & 9.6 & 2.4 & 15.82 & 3.19 & 35.52 & 23.68 \\
\hline $24 \mathrm{~S}$ & 13.64 & 13.2 & 4.4 & 11.74 & 3.3 & $<\mathrm{DL}$ & 12.67 & 8.8 & 13.23 & 12.85 & 17.27 & 2.2 & 10.8 & 17.16 & 11.44 \\
\hline $24 \mathrm{~B}$ & ND & 7.6 & 10.89 & $<\mathrm{DL}$ & 13.25 & 18.54 & $<\mathrm{DL}$ & 29.13 & 9.5 & 7.6 & 1.9 & 9.5 & 8.68 & 5.89 & 3.93 \\
\hline $25 \mathrm{~S}$ & 6.03 & 4.4 & 4.4 & $<\mathrm{DL}$ & 2.2 & $<\mathrm{DL}$ & 2.2 & 12.47 & 4.4 & 12.76 & 16.23 & 13.28 & 9.91 & 57.2 & 38.13 \\
\hline $25 \mathrm{~B}$ & 8.64 & 4.8 & 13.29 & $<\mathrm{DL}$ & $<\mathrm{DL}$ & 7.2 & $<\mathrm{DL}$ & 22.35 & 16.8 & 32.84 & 12.95 & 2.4 & 20.2 & 39.12 & 26.08 \\
\hline $26 \mathrm{~S}$ & 14.76 & 5.4 & 12.35 & $<\mathrm{DL}$ & $<\mathrm{DL}$ & $<\mathrm{DL}$ & 1.8 & 14.28 & 15.93 & 1.8 & 12.36 & 11.29 & 6.17 & 24.12 & 16.08 \\
\hline $26 \mathrm{~B}$ & 5.12 & $<\mathrm{DL}$ & 11.94 & $<\mathrm{DL}$ & 25.14 & 6.2 & 1.6 & 23.47 & 34.73 & 3.2 & 12.93 & 4.8 & 4.61 & 6.88 & 4.59 \\
\hline $27 \mathrm{~S}$ & 3.61 & 9.5 & 3.8 & 1.9 & 5.5 & 5.3 & 6.7 & 14.57 & 3.8 & 5.7 & 1.9 & 5.55 & 8.64 & 36.29 & 25.59 \\
\hline $27 \mathrm{~B}$ & 9.86 & 7.54 & 3.21 & $<\mathrm{DL}$ & $<\mathrm{DL}$ & $<\mathrm{DL}$ & $<\mathrm{DL}$ & 6.8 & 5.1 & 7.95 & 5.84 & 3.92 & 3.06 & 9.52 & 6.35 \\
\hline $28 \mathrm{~S}$ & 4.94 & 5.7 & 11.4 & $<\mathrm{DL}$ & 7.98 & 3.8 & $<\mathrm{DL}$ & 14.57 & 5.7 & 3.8 & 6.94 & 11.4 & 20.84 & 47.31 & 31.54 \\
\hline $28 \mathrm{~B}$ & 4.7 & 26.4 & 16.8 & 3.76 & 3.2 & 11.72 & 2.4 & 12.8 & 7.2 & 4.8 & 9.48 & 5.28 & 29.98 & 27.36 & 18.24 \\
\hline
\end{tabular}

Naph: Naphthalene, Acthy: Acenaphthylene, Ace: Acenaphthene, Phe: Phenanthrene, An: Anthracene, Flu: Fluoranthene, Py: Pyrene, BaA: Benzo(a)anthracene, Chr: Chrysene, BkF: Benzo(k)flouranthene, BaP: Benzo(a)pyrene, BbF: Benzo(b)fluoranthene, InP: Indenopyrene, B(ghi)P: Benzo(g,h,i)perylene, DBA: Dibenz(a,h)anthracene, ND: Not detected, <DL: Lower detection limit, S: Surface, and B: Bottom. 
(c)

\begin{tabular}{|c|c|c|c|c|c|c|c|c|c|c|c|c|c|c|c|}
\hline $\begin{array}{c}\text { Parameter } \\
\text { Site }\end{array}$ & Naph & Acthy & Ace & Phe & Ant & Flu & Pyr & $\mathbf{B a A}$ & Chr & BbF & BkF & BaP & InP & B(ghi)P & DBA \\
\hline $29 \mathrm{~S}$ & 15.64 & 18.4 & 21.94 & $<\mathrm{DL}$ & 2.3 & ND & $<\mathrm{DL}$ & 15.33 & $<\mathrm{DL}$ & $<\mathrm{DL}$ & $<\mathrm{DL}$ & 2.3 & 5.62 & ND & $<\mathrm{DL}$ \\
\hline $29 \mathrm{~B}$ & 27 & 21.6 & 1.8 & 22.13 & 32.95 & 8.4 & 1.8 & 9.6 & 5.4 & 35.71 & 3.1 & 54.37 & 37.23 & 21.78 & 14.52 \\
\hline $30 \mathrm{~S}$ & 8.68 & 13.87 & 4.2 & 6.56 & 4.2 & 7.6 & 1.4 & 12.13 & 5.89 & 1.4 & 16.51 & $<\mathrm{DL}$ & 3.01 & 10.36 & 6.91 \\
\hline $30 \mathrm{~B}$ & 8.1 & 7.5 & 1.5 & 2.54 & 2.89 & 3.79 & 7.6 & 5 & 5.8 & 4.88 & 5.89 & 3.78 & 2.84 & $\mathrm{ND}$ & $<\mathrm{DL}$ \\
\hline $31 \mathrm{~S}$ & 14.44 & 17.1 & 10.82 & $<\mathrm{DL}$ & 8.24 & 2.98 & 1.9 & 8.23 & 3.8 & 7.92 & 3.09 & 1.87 & 1.96 & 11.78 & 7.85 \\
\hline $31 \mathrm{~B}$ & 34.84 & 5.2 & 12.09 & 12.34 & 23.75 & $<\mathrm{DL}$ & 9.5 & 15.6 & 5.2 & 12.34 & 10.64 & $<\mathrm{DL}$ & 5.17 & ND & ND \\
\hline $32 \mathrm{~S}$ & 12.48 & 11.22 & 13.72 & $<\mathrm{DL}$ & $<\mathrm{DL}$ & 3.87 & 10.4 & 5.2 & 13.98 & 12.6 & 2.6 & 7.09 & ND & ND & $<\mathrm{DL}$ \\
\hline $32 \mathrm{~B}$ & 48 & 7.2 & 4.8 & $<\mathrm{DL}$ & 4.86 & 6.78 & 3.96 & 10.4 & 7.2 & 10.92 & 11.28 & 3.87 & 42.44 & 48.96 & 32.64 \\
\hline $33 \mathrm{~S}$ & 11.5 & 25 & 2.5 & $<\mathrm{DL}$ & 6.8 & 5 & $<\mathrm{DL}$ & 3.67 & 16.89 & 5.32 & 7.16 & 2.5 & 4.04 & $\mathrm{ND}$ & ND \\
\hline $33 \mathrm{~B}$ & 17.02 & 18.4 & 23.76 & 2.3 & 11.92 & $<\mathrm{DL}$ & $<\mathrm{DL}$ & 5.37 & 11.82 & 2.3 & $<\mathrm{DL}$ & 6.15 & 3.93 & 42.09 & 28.06 \\
\hline $34 \mathrm{~S}$ & $\mathrm{ND}$ & ND & $<\mathrm{DL}$ & $<\mathrm{DL}$ & $<\mathrm{DL}$ & 2.9 & $<\mathrm{DL}$ & 6.77 & 22.93 & 3.53 & $<\mathrm{DL}$ & 5.98 & 3.65 & 11.02 & 7.35 \\
\hline $34 \mathrm{~B}$ & 4.41 & 5.7 & 5.34 & 9.87 & 3.87 & 7.98 & $<\mathrm{DL}$ & 8.23 & 1.9 & 7.95 & $<\mathrm{DL}$ & $<\mathrm{DL}$ & 2.09 & 6.84 & 4.56 \\
\hline $35 \mathrm{~S}$ & 40.12 & 8.5 & 1.7 & $<\mathrm{DL}$ & $<\mathrm{DL}$ & 2.19 & $<\mathrm{DL}$ & 4.53 & 3.4 & 1.7 & $<\mathrm{DL}$ & $<\mathrm{DL}$ & 4.96 & 10.2 & 6.8 \\
\hline $35 \mathrm{~B}$ & 30.6 & 12 & 3.33 & 4.27 & 5.29 & 3.7 & 5.18 & 13 & 7.5 & 1.5 & 2.17 & 3 & 101.66 & 12.6 & 8.4 \\
\hline $36 \mathrm{~S}$ & 4.42 & 3.4 & $<\mathrm{DL}$ & $\mathrm{ND}$ & ND & $<\mathrm{DL}$ & $\mathrm{ND}$ & ND & ND & 12.67 & $<\mathrm{DL}$ & $<\mathrm{DL}$ & 1.29 & $\mathrm{ND}$ & $<\mathrm{DL}$ \\
\hline $36 \mathrm{~B}$ & 75.36 & 24 & 23.14 & $<\mathrm{DL}$ & 3.76 & $<\mathrm{DL}$ & $<\mathrm{DL}$ & 3.91 & 12.67 & 8.98 & 4.99 & 2.4 & 68.72 & 11.02 & 7.34 \\
\hline $37 \mathrm{~S}$ & ND & 5.5 & 7.8 & 9.6 & 8.5 & 2.4 & $<\mathrm{DL}$ & 16.2 & 9.6 & 2.4 & $<\mathrm{DL}$ & $<\mathrm{DL}$ & ND & 9.12 & 6.08 \\
\hline $37 \mathrm{~B}$ & 4.75 & 17.5 & 10 & 12.95 & 3.65 & 2.5 & $<\mathrm{DL}$ & 6.67 & 9.37 & 8.64 & 13.24 & 2.5 & 181.85 & 22.25 & 14.83 \\
\hline $38 \mathrm{~S}$ & 16.2 & 5.4 & 8.1 & 2.7 & 11.9 & 4.5 & 2.7 & 7.2 & 35.1 & 5.4 & 2.7 & $<\mathrm{DL}$ & ND & $\mathrm{ND}$ & $<\mathrm{DL}$ \\
\hline $38 \mathrm{~B}$ & 3.78 & ND & $<\mathrm{DL}$ & 2.7 & $<\mathrm{DL}$ & $<\mathrm{DL}$ & $<\mathrm{DL}$ & $<\mathrm{DL}$ & $<\mathrm{DL}$ & 2.83 & 12.86 & 3.98 & 20.33 & 28.35 & 18.9 \\
\hline $39 \mathrm{~S}$ & 28.5 & 7.5 & 7.5 & 25 & 2.5 & $<\mathrm{DL}$ & 7.98 & 39.17 & 7.12 & 2.5 & 14.5 & 7.5 & 4.33 & 24.75 & 16.5 \\
\hline 39B & 36.48 & 7.2 & 5.94 & 3.79 & $<\mathrm{DL}$ & 2.4 & ND & 5.6 & 4.8 & 7.68 & 4.8 & $<\mathrm{DL}$ & 22.8 & 14.4 & 9.6 \\
\hline
\end{tabular}

Naph: Naphthalene, Acthy: Acenaphthylene, Ace: Acenaphthene, Phe: Phenanthrene, An: Anthracene, Flu: Fluoranthene, Py: Pyrene, BaA: Benzo(a)anthracene, Chr: Chrysene, BkF: Benzo(k)flouranthene, BaP: Benzo(a)pyrene, BbF: Benzo(b)fluoranthene, InP: Indenopyrene, B(ghi)P: Benzo(g,h,i)perylene, DBA: Dibenz(a,h)anthracene, ND: Not detected, $<$ DL: Lower detection limit, S: Surface, and B: Bottom.

(d)

\begin{tabular}{|c|c|c|c|c|c|c|c|c|c|c|c|c|c|c|c|}
\hline $\begin{array}{c}\text { Parameter } \\
\text { Site } \\
\end{array}$ & Naph & Acthy & Ace & Phe & Ant & Flu & Pyr & $\mathbf{B a A}$ & Chr & $\mathbf{B b F}$ & BkF & $\mathrm{BaP}$ & InP & B(ghi)P & DBA \\
\hline $40 \mathrm{~S}$ & 5.02 & 8.8 & 2.2 & 11 & 3.8 & 7.93 & 8.3 & 6.6 & 3.98 & 17.6 & 13.2 & 12.67 & 6.61 & 20.9 & 13.93 \\
\hline $40 \mathrm{~B}$ & 15.84 & 11.98 & 23.45 & 13.2 & 12.34 & 11 & 11.43 & 22 & 5.67 & 6.6 & 12.78 & 23.89 & 5.64 & 4.4 & 2.93 \\
\hline $41 \mathrm{~S}$ & 23.56 & 3.98 & 2.98 & 5.7 & 21.67 & 3.6 & 3.29 & 8.87 & 4.67 & 3.86 & 1.97 & 6.98 & 5.75 & 1.33 & 0.89 \\
\hline $41 \mathrm{~B}$ & 21.08 & 5.98 & 4.67 & 6.8 & 3.4 & 8.5 & 3.87 & 29.47 & 3.4 & 18.7 & 11.45 & 7.65 & 9.22 & 24.82 & 16.55 \\
\hline $42 \mathrm{~S}$ & 6.8 & 5.1 & 3.4 & 3.4 & 3.4 & 3.4 & 4.67 & 5.67 & 3.98 & 6.8 & 0 & 2.97 & 4.22 & 15.64 & 10.43 \\
\hline $42 \mathrm{~B}$ & 11.78 & 7.6 & 3.8 & 6.41 & 5.7 & 38 & 2.68 & 5.07 & 1.9 & 7.6 & 3.8 & 9.5 & 5.4 & 9.31 & 6.21 \\
\hline $43 \mathrm{~S}$ & 24.82 & 8.5 & 7.93 & 5.1 & 2.86 & 8.2 & 9.57 & 9.63 & 8.5 & 10.2 & 12.29 & 6.94 & 5.04 & 11.05 & 7.37 \\
\hline $43 B$ & 9.5 & 5.7 & 5.7 & 7.6 & 5.7 & 7.6 & 3.8 & 10.77 & 5.7 & 11.4 & 5.7 & 20.9 & 4.38 & 32.49 & 21.66 \\
\hline $44 \mathrm{~S}$ & 23.52 & 3.29 & 7.95 & 3.76 & 2.4 & 6.3 & 2.4 & 10.4 & 4.8 & 4.8 & 7.98 & 3.18 & 8.45 & 24.72 & 16.48 \\
\hline $44 \mathrm{~B}$ & 35.36 & 7.94 & 10.4 & 4.57 & 2.6 & 13 & 7.8 & 13 & 10.4 & 26 & 15.6 & 23.4 & 14.17 & 25.74 & 17.16 \\
\hline $45 \mathrm{~S}$ & 11 & 5.9 & 12.5 & 23.7 & 3.9 & 1.92 & 5.3 & 2.78 & 5.94 & 8.8 & 3.94 & 2.2 & 3.72 & 10.78 & 7.19 \\
\hline $45 \mathrm{~B}$ & 27.14 & 4.6 & 6.9 & 7.94 & 4.6 & 13.8 & $<\mathrm{DL}$ & 9.2 & 23 & 4.6 & 13.8 & 9.2 & 14.55 & 92.23 & 61.49 \\
\hline $46 \mathrm{~S}$ & 4.37 & 3.99 & 10.4 & $<\mathrm{DL}$ & 11.3 & 2.6 & 8.91 & 7.8 & 18.2 & 7.8 & 11.84 & 7.94 & 7.75 & 26.26 & 17.51 \\
\hline $46 \mathrm{~B}$ & 5.2 & 3.29 & 23.94 & 7.5 & 4.68 & 6.88 & 6.48 & 10.83 & 7.96 & 17.5 & 10.19 & 4.96 & 9.21 & 23.5 & 15.67 \\
\hline $47 \mathrm{~S}$ & 24.48 & 6.93 & 8.74 & 9.39 & 4.8 & 8.49 & 3.92 & 4.38 & 2.4 & 9.88 & 8.97 & 4.83 & 5.98 & 37.44 & 24.96 \\
\hline 47B & 7.92 & 6.59 & 10.8 & 3.02 & 3.6 & 10.8 & 7.86 & 21 & 5.4 & 14.4 & 18.75 & 13.56 & 10.78 & 28.44 & 18.96 \\
\hline $48 \mathrm{~S}$ & 12.6 & 8.95 & 3.6 & 21.83 & 9.48 & 7.49 & 5.48 & 9.6 & 4.94 & 12.39 & 6.59 & 5.4 & 6.44 & 27 & 18 \\
\hline $48 \mathrm{~B}$ & 24.24 & 10.8 & 4.8 & 26.4 & 5.94 & 7.2 & 8.75 & 13.6 & 3.6 & 10.29 & 8.4 & 13.2 & 3.02 & 15.48 & 10.32 \\
\hline $49 \mathrm{~S}$ & 25.22 & 9.87 & 3.55 & 6.5 & 2.6 & 3.9 & 5.9 & 8.67 & 8.96 & 9.1 & 8.77 & 4.39 & 4.62 & 26.65 & 17.77 \\
\hline $49 \mathrm{~B}$ & 11 & 4.76 & 8.65 & 12.5 & 2.2 & 13.2 & 6.95 & 11.73 & 9.76 & 15.4 & 17.3 & 6.6 & 13.02 & 37.62 & 25.08 \\
\hline
\end{tabular}

Naph: Naphthalene, Acthy: Acenaphthylene, Ace: Acenaphthene, Phe: Phenanthrene, An: Anthracene, Flu: Fluoranthene, Py: Pyrene, BaA: Benzo(a)anthracene, Chr: Chrysene, BkF: Benzo(k)flouranthene, BaP: Benzo(a)pyrene, BbF: Benzo(b)fluoranthene, InP: Indenopyrene, B(ghi)P: Benzo(g,h,i)perylene, DBA: Dibenz(a,h)anthracene, ND: Not detected, <DL: Lower detection limit, S: Surface, and B: Bottom. 


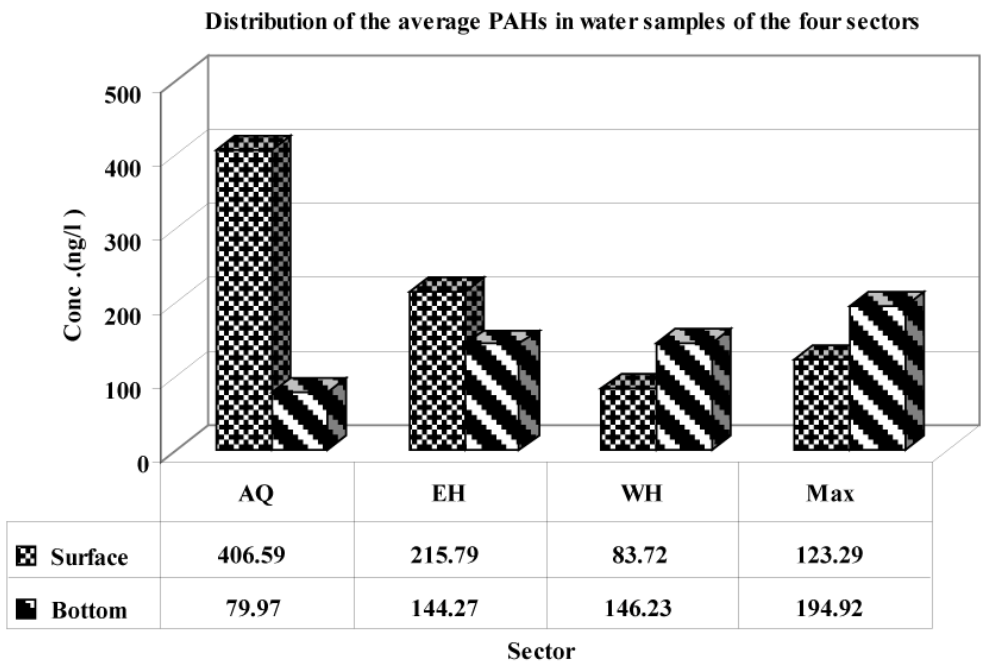

Figure 2. Variation of average PAHs concentrations (ng/l) in surface and bottom water samples of the four areas.

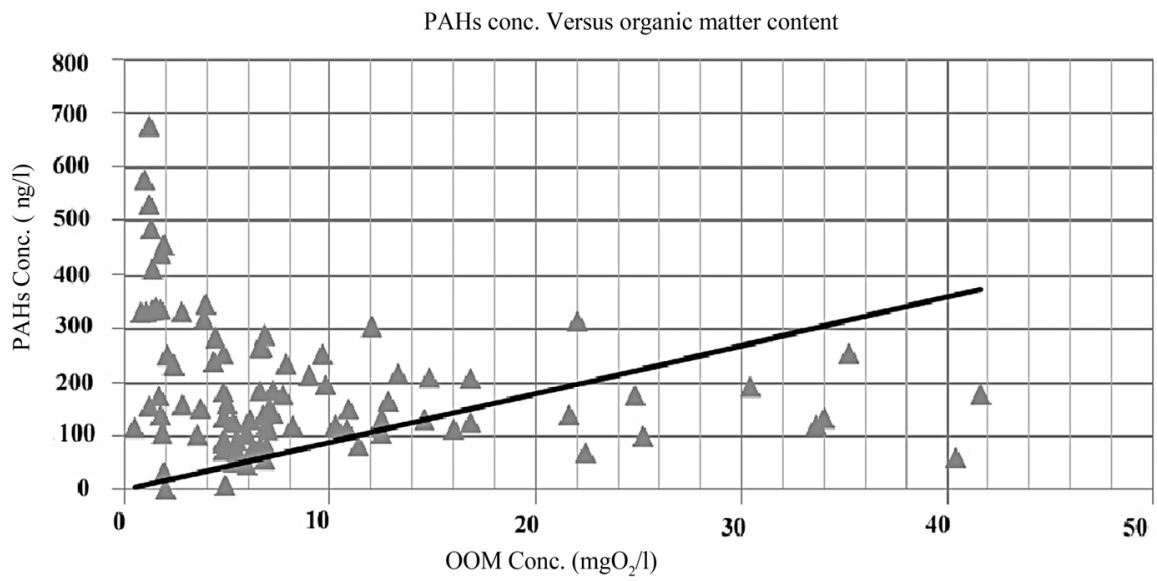

Figure 3. Relationship between total PAH concentrations (ng/l) and $\mathrm{OOM}\left(\mathrm{mgO}_{2} / \mathrm{l}\right)$ of the studied area.

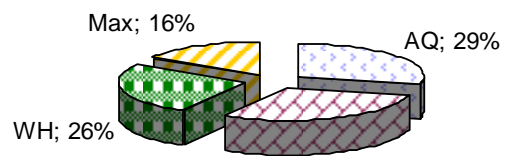

$\mathrm{EH} ; 29 \%$

(a)

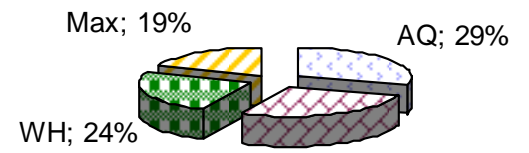

$\mathrm{EH} ; 28 \%$

(b)

Figure 4. A comparison of the average combustion\% of PAHs in: (a) surface and (b) bottom waters in the four areas.

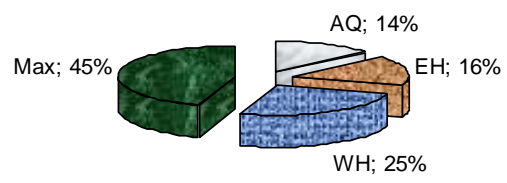

(a)

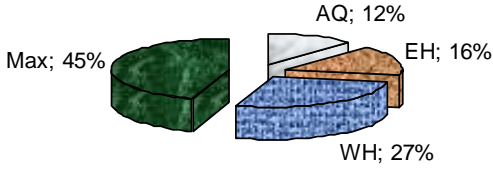

(b)

Figure 5. A comparison of the average fossil \% of PAHs in: (a) surface and (b) bottom water in the four areas.

water samples collected from the studied area during 2010. The average values of water salinity at different areas revealed that the salinity decreased in the order: $\mathrm{WH}>\mathrm{EH}>\mathrm{AQ}>\mathrm{Max}$, the average $\mathrm{pH}$ values are decreasing in the order; $\mathrm{AQ}>\mathrm{WH}>\mathrm{EH}>$ Max. Concen- tration of DO was decreasing in the order; $\mathrm{EH}>\mathrm{AQ}>\mathrm{W}$ $\mathrm{H}>\mathrm{Max}$, in case of OOM; it was noticed that the general trend showed that AQ exhibited the lowest content of OOM compared to other areas and the order was decreasing from: $\mathrm{EH}>\mathrm{Max}>\mathrm{WH}>\mathrm{AQ}$. 
Table 2. Comparison of PAHs concentrations (ng/l) in water recorded in the present study with areas of the surroundings.

\begin{tabular}{|c|c|c|}
\hline Name of Site & Range (ng. $\left.\right|^{-1}$ ) & Reference \\
\hline Abu-Qir Bay, Alexandria, Egypt & $127.17-456.19$ & \multirow{4}{*}{ Present study } \\
\hline Eastern Harbour, Alexandria, Egypt & $75.10-286.02$ & \\
\hline Western Harbour, Alexandria, Egypt & $29.31-292.72$ & \\
\hline El-Max Bay, Alexandria, Egypt & $107.95-209.0$ & \\
\hline Deep Bay, South China & $69.4-24.7$ & [3] \\
\hline Tonghui River of Beijing, China & $192.5-2651$ & [9] \\
\hline Red Sea Coast, Egypt & $400-96,450$ & [25] \\
\hline Alexandria Coast, Egypt & $13-120$ & [26] \\
\hline Alexandria Coast, Egypt & 117.5 - 564 & [27] \\
\hline Brighton Marina, UK & $<2$ and 11,400 & [40] \\
\hline Jiulong River Estuary and Western Xiamen Sea, China & $6960-26,900$ & [43] \\
\hline Aquatic Environment, El-Menofiya Governorate, Egypt & $226.9-1492.2$ & [44] \\
\hline Creek, Niger Delta region & $24,390-283,600$ & [45] \\
\hline Bahía Blanca Estuary, Argentina & ND - 4000 & [46] \\
\hline Gerlache Inlet Sea, Antarctica & $5.27-9.43$ & [47] \\
\hline
\end{tabular}

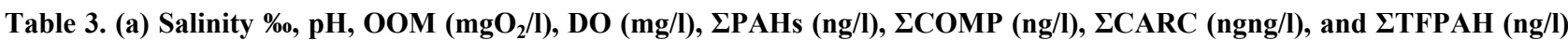
in water samples collected From Abou Qir Bay (AQ) during 2010; (b) Salinity \%o, pH, OOM (mgO $/$ /), DO (mg/l), $\Sigma$ PAHs

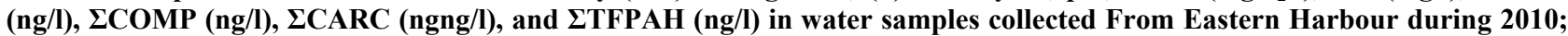

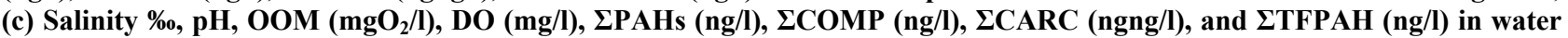

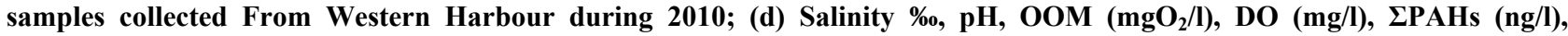

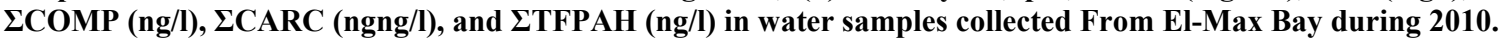

(a)

\begin{tabular}{|c|c|c|c|c|c|c|c|c|c|c|c|c|c|c|c|c|}
\hline \multirow{2}{*}{$\begin{array}{c}\text { Parameter } \\
\text { Site }\end{array}$} & \multicolumn{2}{|c|}{ Salinity \%o } & \multicolumn{2}{|c|}{ pH } & \multicolumn{2}{|c|}{$\mathrm{OOM}\left(\mathrm{mgO}_{2} / \mathrm{l}\right)$} & \multicolumn{2}{|c|}{ DO (mg/l) } & \multicolumn{2}{|c|}{ इPAHs (ng/l) } & \multicolumn{2}{|c|}{$\Sigma C O M P(n g / l)$} & \multicolumn{2}{|c|}{ इCARC (ng/l) } & \multicolumn{2}{|c|}{ इTFPAH (ng/l) } \\
\hline & $\mathrm{S}$ & $\mathrm{B}$ & $\mathrm{S}$ & $\mathrm{B}$ & $\mathrm{S}$ & B & $\mathrm{S}$ & $\mathrm{B}$ & $\mathrm{S}$ & $\mathrm{B}$ & $\mathrm{S}$ & $\mathrm{B}$ & $\mathrm{S}$ & $\mathrm{B}$ & $\mathrm{S}$ & $\mathrm{B}$ \\
\hline 1 & 38.7 & NR & 8.48 & NR & 1.35 & NR & 2.93 & NR & 411.17 & NR & 327.17 & NR & 206.57 & NR & 67.2 & NR \\
\hline 2 & 38.5 & 38.5 & 8.65 & 8.71 & 1.19 & 1.98 & 4.55 & 5.53 & 531.16 & 2.21 & 427.16 & 2.21 & 323.42 & 2.21 & 91 & $\mathrm{NC}$ \\
\hline 3 & 8.5 & 38.4 & 8.22 & 8.57 & 1.9 & 1.19 & 6.18 & 4.23 & 456.14 & 156.6 & 409.34 & 156.6 & 280.46 & 78.4 & 41.4 & $\mathrm{NC}$ \\
\hline 4 & 35.1 & 38.7 & 8.9 & 8.5 & 1.27 & 1.66 & 5.85 & 3.87 & 484.98 & 175.56 & 431.78 & 171.16 & 224.11 & 109.56 & 45.6 & 2.2 \\
\hline 5 & 34.7 & 38.6 & 9.08 & 8.51 & 1.74 & 2.37 & 6.94 & 4.52 & 440.5 & 232.54 & 325.5 & 196.54 & 210.96 & 142.3 & 98.9 & 33.6 \\
\hline 6 & 36.6 & 38.6 & 9.01 & 8.75 & 1.19 & 1.82 & 7.1 & 5.16 & 677.25 & 106.81 & 635.65 & 104.51 & 285.69 & 70.7 & 33.8 & $\mathrm{NC}$ \\
\hline 7 & 38 & 38.5 & 8.87 & 8.73 & 0.95 & 1.9 & 5.97 & 3.55 & 578.03 & .61 & 498.03 & 29.41 & 410.03 & 14.33 & 65 & $\mathrm{NC}$ \\
\hline 8 & 38.4 & 38.6 & 8.72 & 8.69 & 0.79 & 1.74 & 6.45 & 4.52 & 330.15 & 139.59 & 238.95 & 137.79 & 161.24 & 83.07 & 87.4 & $\mathrm{NC}$ \\
\hline 9 & 38.4 & 38.5 & 8.75 & 8.74 & 1.35 & 2.37 & 4.52 & 3.23 & 333.83 & 235.12 & 292.03 & 193.52 & 231.8 & 179.06 & 38 & 36.8 \\
\hline 10 & 38.4 & 38.4 & 8.81 & 8.81 & 1.03 & 0.47 & 4.52 & 6.45 & 330.79 & 116.57 & 265.99 & 78.57 & 231.98 & 53.11 & 55.8 & 36.1 \\
\hline 11 & 38.9 & NR & 8.98 & NR & 2.77 & NR & 3.23 & NR & 330.33 & NR & 224.93 & NR & 167.13 & NR & 102 & NR \\
\hline 12 & 28.7 & NR & 8.98 & NR & 1.5 & NR & 5.48 & NR & 339.27 & NR & 286.47 & NR & 239.83 & NR & 45.6 & NR \\
\hline 13 & 33.8 & NR & 8.72 & NR & 2.06 & NR & 4.19 & NR & 252.14 & NR & 190.94 & NR & 144.53 & NR & 52.7 & NR \\
\hline 14 & 35 & NR & 9.07 & NR & 1.74 & NR & 3.55 & NR & 335.5 & NR & 265.3 & $\mathrm{NI}$ & 203.79 & NR & 62.4 & NR \\
\hline 15 & 38 & NR & 8.68 & NR & 6.65 & NR & 3.87 & NR & 267.63 & NR & 218.23 & NR & 164.65 & NR & 41.8 & NR \\
\hline
\end{tabular}

OOM: Oxidizable organic matter, DO: Dissolved oxygen, Av: average, Max: maximum, Min: minimum, NR: Not collected, NC: Not calculated, PAHs: Sum of aromatic hydrocarbons, $\Sigma$ CARC: Sum of BaA + BbF + BkF + BaP + DBA + InP, $\Sigma$ TFPAH: Sum Acenaphthylene + Acenaphthene, $\Sigma$ COMP: Sum of all PAHs except those calculated for $\Sigma$ TFPAH.

(b)

\begin{tabular}{|c|c|c|c|c|c|c|c|c|c|c|c|c|c|c|c|c|}
\hline \multirow{2}{*}{$\begin{array}{c}\text { Parameter } \\
\text { Site }\end{array}$} & \multicolumn{2}{|c|}{ Salinity \%o } & \multicolumn{2}{|c|}{ pH } & \multicolumn{2}{|c|}{$\mathrm{OOM}_{\left(\mathrm{mgO}_{2} / \mathrm{l}\right)}$} & \multicolumn{2}{|c|}{ DO (mg/l) } & \multicolumn{2}{|c|}{ इPAHs (ng/l) } & \multicolumn{2}{|c|}{$\Sigma$ COMP (ng/l) } & \multicolumn{2}{|c|}{$\Sigma$ CARC (ng/l) } & \multicolumn{2}{|c|}{ इTFPAH (ng/l) } \\
\hline & $\mathbf{S}$ & B & $\mathbf{S}$ & B & $\mathbf{S}$ & B & $\mathbf{S}$ & B & $\mathbf{S}$ & B & $\mathbf{S}$ & B & $\mathbf{S}$ & B & $\mathbf{S}$ & B \\
\hline 16 & 39.1 & 39.7 & 7.76 & 7.78 & 3.84 & 24.8 & 6.13 & 5.24 & 317.2 & 176.93 & 274.45 & 143.39 & 173.45 & 119.21 & 35.25 & 28.34 \\
\hline 17 & 38.9 & 39.4 & 7.63 & 7.56 & 22 & 33.6 & 3.15 & 4.6 & 313.74 & 121.2 & 262.26 & 104.1 & 177.56 & 72.18 & 49.28 & 13.3 \\
\hline 18 & 39.1 & 39.2 & 7.5 & 7.56 & 6.8 & 21.6 & 3.23 & 3.79 & 288.94 & 140.42 & 187.06 & 113.7 & 166.18 & 93.86 & 96.48 & 25.12 \\
\hline 19 & 39.1 & 39.4 & 7.57 & 7.65 & 9.6 & 34 & 3.63 & 5.16 & 253.59 & 134.05 & 202.89 & 105.23 & 155.49 & 49.13 & 47.7 & 24.42 \\
\hline 20 & 39.1 & 39.3 & 7.64 & 7.71 & 12 & 22.4 & 4.84 & 4.76 & 304.03 & 70.7 & 250.51 & 58.38 & 132.19 & 42.22 & 43.92 & 10.72 \\
\hline 21 & 39.2 & 39.6 & 7.75 & 7.71 & 4.4 & 16.8 & 5.81 & 4.19 & 283.71 & 126.59 & 205 & 95.84 & 131.73 & 63.74 & 75.31 & 29.25 \\
\hline 22 & 39.3 & 39.5 & 7.72 & 7.78 & 2.8 & 4.8 & 5.32 & 4.68 & 158.77 & 135.32 & 122.45 & 116.89 & 74.61 & 91.43 & 31.52 & 16.53 \\
\hline 23 & 39.3 & 39.4 & 7.82 & 7.86 & 25.2 & 7.2 & 6.05 & 5.81 & 100.95 & 142.94 & 88.45 & 118.7 & 64.2 & 75.98 & 10 & 21.84 \\
\hline 24 & 39.3 & 39.3 & 7.74 & 7.81 & 10.8 & 16.8 & 6.13 & 4.76 & 113.78 & 209.46 & 84.96 & 198.06 & 61.2 & 180.77 & 24.42 & 9.5 \\
\hline 25 & 39.5 & 39.7 & 7.75 & 7.8 & 30.4 & 7.2 & 5.24 & 5.97 & 192.96 & 187.03 & 151.82 & 175.51 & 88.02 & 112.39 & 38.94 & 11.52 \\
\hline 26 & 39.5 & 39.7 & 7.59 & 7.69 & 4.8 & 16 & 5.89 & 5.08 & 88.83 & 114.66 & 72.45 & 107.3 & 42.93 & 92.42 & 16.38 & 5.76 \\
\hline 27 & 39.5 & 39.6 & 7.68 & 7.71 & 41.6 & 40.4 & 4.68 & 5.65 & 178.2 & 61.37 & 143.05 & 53.04 & 97.07 & 38.42 & 31.35 & 8.33 \\
\hline 28 & 39.4 & 39.7 & 7.69 & 7.62 & 14.8 & 35.2 & 4.68 & 3.39 & 210.6 & 254.83 & 166.9 & 183.31 & 110.09 & 131.95 & 41.8 & 66.72 \\
\hline
\end{tabular}

OOM: Oxidizable organic matter, DO: Dissolved oxygen, Av: average, Max: maximum, Min: minimum, NR: Not collected, NC: Not calculated, PAHs: Sum of aromatic hydrocarbons, $\Sigma$ CARC: Sum of BaA + BbF + BkF + BaP + DBA + InP, $\Sigma$ TFPAH: Sum Acenaphthylene + Acenaphthene, $\Sigma$ COMP: Sum of all PAHs except those calculated for $\Sigma$ TFPAH. 
(c)

\begin{tabular}{|c|c|c|c|c|c|c|c|c|c|c|c|c|c|c|c|c|}
\hline \multirow{2}{*}{$\begin{array}{c}\text { Parameter } \\
\text { Site }\end{array}$} & \multicolumn{2}{|c|}{ Salinity \%o } & \multicolumn{2}{|c|}{ pH } & \multicolumn{2}{|c|}{$\mathrm{OOM}\left(\mathrm{mgO}_{2} / \mathrm{l}\right)$} & \multicolumn{2}{|c|}{ DO (mg/l) } & \multicolumn{2}{|c|}{ ¿PAHs (ng/l) } & \multicolumn{2}{|c|}{$\Sigma$ ¿OMP (ng/l) } & \multicolumn{2}{|c|}{ इCARC (ng/l) } & \multicolumn{2}{|c|}{ इTFPAH (ng/l) } \\
\hline & $\mathbf{S}$ & B & $\mathbf{S}$ & B & $\mathbf{S}$ & B & $\mathbf{S}$ & B & $\mathbf{S}$ & B & $\mathbf{S}$ & B & $\mathbf{S}$ & B & $\mathbf{S}$ & B \\
\hline 29 & 40.8 & 42.2 & 7.77 & 7.75 & 4.8 & 6.56 & 3.99 & 3.72 & 94.97 & 185.29 & 64.15 & 144.79 & & 114.01 & 28.52 & 36.9 \\
\hline 30 & 35 & 40.3 & 72 & 7.7 & & & & & & & & & & & & \\
\hline & & 40. & 71 & 7.8 & & & & & & & & & & & & \\
\hline & & & & & & & & & & & & & & & & \\
\hline & 4 & & 77 & 7.7 & & & & & & & & & & & 33 & \\
\hline 34 & 0.8 & $40 .{ }^{2}$ & 7.75 & 7.76 & & & & 5. & 54.23 & & & & & & $\mathrm{~N}$ & 29.64 \\
\hline 35 & 40.4 & 40.1 & 7.78 & 7.76 & & & & 4.9 & 72.47 & & & & 26. & & 30. & 28.8 \\
\hline 36 & 41 & 41.3 & 7.76 & 7.78 & 4.88 & 3.92 & 4.43 & 4.19 & 9.89 & & 4.28 & 278.81 & 4.28 & 163.85 & 5.61 & 64.08 \\
\hline 37 & 40.5 & 41.2 & 7.8 & 7.79 & 5.36 & 3.68 & 4.74 & 6.33 & 54.72 & 152.37 & 45.12 & 96.12 & 24 & 68.87 & 4.8 & 51.25 \\
\hline 38 & 40.8 & 41.2 & 7.81 & 7.81 & 5.92 & 6.56 & 6.05 & 5.53 & 105.3 & 81.7 & 78.3 & 77.11 & 37.8 & 48.76 & 21.6 & 1.89 \\
\hline 39 & 40.5 & 40.6 & 7.77 & 7.74 & 4.32 & 5.28 & 5.06 & 4.19 & 240.15 & 126.42 & 183.4 & 91.38 & 151.15 & 69.78 & 29.25 & 32.64 \\
\hline
\end{tabular}

OOM: Oxidizable organic matter, DO: Dissolved oxygen, Av: average, Max: maximum, Min: minimum, NR: Not collected, NC: Not calculated, PAHs: Sum of

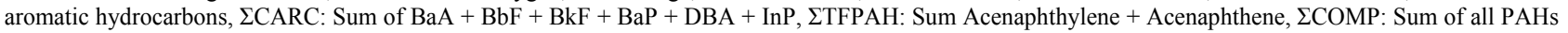
except those calculated for $\Sigma$ TFPAH.

(d)

\begin{tabular}{|c|c|c|c|c|c|c|c|c|c|c|c|c|c|c|c|c|}
\hline \multirow{2}{*}{$\begin{array}{c}\text { Parameter } \\
\text { Site }\end{array}$} & \multicolumn{2}{|c|}{ Salinity \%o } & \multicolumn{2}{|c|}{ pH } & \multicolumn{2}{|c|}{$\mathrm{OOM}\left(\mathrm{mgO}_{2} / \mathrm{l}\right)$} & \multicolumn{2}{|c|}{ DO (mg/l) } & \multicolumn{2}{|c|}{ इPAHs (ng/l) } & \multicolumn{2}{|c|}{$\Sigma C O M P(n g / l)$} & \multicolumn{2}{|c|}{ ICARC (ng/l) } & \multicolumn{2}{|c|}{ ¿TFPAH (ng/l) } \\
\hline & $\mathbf{S}$ & B & $\mathbf{S}$ & B & $\mathbf{S}$ & B & $\mathbf{S}$ & B & $\mathbf{S}$ & B & $\mathbf{S}$ & B & $\mathbf{S}$ & B & $\mathbf{S}$ & B \\
\hline 40 & 36.2 & 40 & 7.27 & 7.3 & 10.88 & 7.68 & 3.87 & 3.64 & 151.6 & 177.61 & 102.32 & 141.09 & 74.82 & 114.69 & 36.08 & 18.92 \\
\hline 41 & 23.6 & 38.5 & 7.04 & 7.33 & 11.36 & 8.96 & 3.56 & 4.03 & 83.51 & 213.61 & 60.33 & 182.67 & 51.4 & 144.25 & 15.58 & 20.74 \\
\hline 42 & 9.7 & 38 & 6.83 & 7.13 & 5.44 & 12.48 & 1.11 & 4.27 & 93.77 & 132.39 & 75.07 & 107.5 & 50.93 & 56.39 & 11.9 & 17.29 \\
\hline 43 & 25.8 & 38.5 & 6.94 & 7.14 & 8.16 & 12.8 & 2.93 & 3.87 & 120.24 & 165.7 & 90.83 & 136.25 & 67.88 & 86.66 & 22.61 & 16.15 \\
\hline 44 & 36.3 & 38.6 & 7.17 & 7.29 & 6.72 & 7.84 & 4.03 & 4.19 & 139.78 & 235.56 & 104.02 & 199.68 & 69.7 & 142.74 & 26.16 & 30.68 \\
\hline 45 & 35.7 & 38.7 & 7.12 & 7.3 & 12.48 & 6.56 & 2.21 & 4.19 & 107.32 & 266 & 84.22 & 229.43 & 64.64 & 100.4 & 14.3 & 25.07 \\
\hline 46 & 28.8 & 38.8 & 7.1 & 7.29 & 7.04 & 4.8 & 2.69 & 5.14 & 152 & 182.93 & 114.56 & 131.93 & 64.9 & 90.93 & 34.84 & 41 \\
\hline 47 & 27.4 & 38.6 & 7.07 & 7.27 & 14.56 & 13.28 & 3.16 & 5.38 & 131.23 & 217.76 & 106.99 & 186.8 & 62.35 & 140.36 & 17.04 & 18.36 \\
\hline 48 & 30.8 & 38.9 & 7.08 & 7.27 & 10.24 & 4.96 & 3.8 & 5.69 & 121.79 & 160.84 & 99.29 & 104.32 & 61.49 & 75.64 & 11.7 & 27.72 \\
\hline 49 & 34.9 & 38.9 & 7.2 & 7.3 & 6.08 & 9.76 & 4.66 & 5.53 & 131.7 & 196.77 & 94.39 & 171.47 & 58.64 & 103.05 & 28.21 & 20.9 \\
\hline
\end{tabular}

OOM: Oxidizable organic matter, DO: Dissolved oxygen, Av: average, Max: maximum, Min: minimum, NR: Not collected, NC: Not calculated, PAHs: Sum of aromatic hydrocarbons, $\Sigma$ CARC: Sum of BaA + BbF + BkF + BaP + DBA + InP, $\Sigma$ TFPAH: Sum Acenaphthylene + Acenaphthene, $\Sigma$ COMP: Sum of all PAHs except those calculated for $\Sigma$ TFPAH.

Table 4. Correlation coefficient matrix for water individual PAHs and some physic-chemical parameters $(\mathrm{n}=92)$.

\begin{tabular}{|c|c|c|c|c|c|c|c|c|c|c|c|c|c|c|c|c|c|c|c|}
\hline Cpd & Naph & Acthy & Ace & Phe & Ant & Flu & Pyr & $\mathbf{B a A}$ & Chr & BbF & BkF & $\mathrm{BaP}$ & InP & $\begin{array}{c}\text { B(ghi) } \\
\text { P }\end{array}$ & DBA & OOM & pH & DO & РAH \\
\hline Naph & 1.000 & & & & & & & & & & & & & & & & & & \\
\hline Acthy & 0.212 & 1.000 & & & & & & & & & & & & & & & & & \\
\hline Ace & 0.274 & 0.312 & 1.000 & & & & & & & & & & & & & & & & \\
\hline Phe & 0.034 & -0.017 & 0.001 & 1.000 & & & & & & & & & & & & & & & \\
\hline Ant & 0.068 & 0.188 & 0.144 & 0.205 & 1.000 & & & & & & & & & & & & & & \\
\hline Flu & -0.032 & $2-0.197$ & -0.013 & 0.147 & 0.155 & 1.000 & & & & & & & & & & & & & \\
\hline Pyr & 0.183 & 0.158 & 0.300 & 0.073 & 0.098 & 0.067 & 1.000 & & & & & & & & & & & & \\
\hline $\mathrm{BaA}$ & 0.012 & 0.024 & 0.163 & 0.140 & 0.118 & 0.212 & 0.212 & 1.000 & & & & & & & & & & & \\
\hline $\mathrm{Chr}$ & 0.020 & 0.059 & 0.064 & -0.011 & 0.228 & 0.244 & 0.055 & 0.227 & 1.000 & & & & & & & & & & \\
\hline $\mathrm{BbF}$ & -0.013 & -0.188 & -0.070 & 0.065 & 0.113 & $0.568^{* *}$ & 0.034 & 0.288 & $0.482^{* *}$ & * 1.000 & & & & & & & & & \\
\hline $\mathrm{BkF}$ & -0.037 & $7-0.187$ & -0.006 & 0.004 & 0.102 & $0.472^{* *}$ & 0.104 & 0.069 & 0.242 & $0.554^{* *}$ & * 1.000 & & & & & & & & \\
\hline $\mathrm{BaP}$ & -0.095 & -0.221 & -0.112 & 0.167 & 0.194 & 0.652 & -0.028 & 0.209 & 0.101 & 0.641 & 0.606 & 1.000 & & & & & & & \\
\hline $\mathrm{InP}$ & 0.168 & 0.200 & 0.025 & 0.011 & 0.013 & 0.163 & -0.059 & 0.106 & 0.136 & 0.254 & 0.044 & 0.119 & 1.000 & & & & & & \\
\hline $\mathrm{B}$ (ghi)P & 0.040 & -0.187 & -0.089 & 0.072 & -0.060 & $0.387^{*}$ & -0.073 & 0.190 & 0.053 & $0.322^{*}$ & 0.151 & $0.455^{* *}$ & 0.211 & 1.000 & & & & & \\
\hline DBA & 0.040 & -0.187 & -0.090 & 0.071 & -0.060 & $0.387^{*}$ & -0.073 & 0.190 & 0.053 & $0.321^{*}$ & 0.151 & $0.454^{* *}$ & 0.211 & $1.000^{* * *}$ & 1.000 & & & & \\
\hline OOM & -0.023 & 0.240 & 0.144 & -0.069 & 0.012 & -0.133 & 0.195 & 0.029 & -0.088 & -0.256 & -0.202 & -0.279 & -0.158 & -0.138 & -0.135 & 51.000 & & & \\
\hline $\mathrm{pH}$ & -0.229 & -0.298 & -0.261 & -0.116 & -0.026 & $0.359^{*}$ & -0.223 & 0.143 & 0.088 & $0.458^{* *}$ & $0.396^{* *}$ & $0.516^{* *}$ & 0.177 & $0.355^{*}$ & $0.354^{*}$ & ${ }^{*}-0.400$ & 1.000 & & \\
\hline DO & -0.062 & -0.267 & -0.101 & -0.064 & -0.083 & 0.151 & -0.046 & 0.101 & 0.028 & 0.106 & 0.281 & 0.209 & 0.102 & 0.195 & 0.195 & -0.053 & $0.325^{*}$ & 1.000 & \\
\hline PAH & 0.127 & -0.050 & 0.097 & 0.131 & 0.109 & 0.650 & 0.038 & $0.362^{*}$ & $0.313^{*}$ & $=0.640$ & 0.642 & $\underset{* * *}{0.721}$ & 0.299 & 0.576 & ${ }_{* * *}^{0.575}$ & -0.2370 & $0.494^{* *}$ & 0.205 & 1.000 \\
\hline
\end{tabular}

Correlations are significant at ${ }^{*} \mathrm{p}<0.05$ (low significant), ${ }^{* *} \mathrm{p}<0.01$ (moderate significant) and ${ }^{* * *} \mathrm{p}<0.001$ (high significant). 


\section{Conclusion}

The present comprehensive study represents the detailed study of the distribution and correlation between physicochemical parameters and PAHs in 92 water samples collected from four semi-closed areas along the Alexandria coast of Egypt. The studied samples were less contaminated by petroleum hydrocarbons. The average concentration of PAHs in eastern-southern Mediterranean Sea waters are shown to be substantially lower in the present study to those in most areas of urbanized estuaries and coastal areas in the other countries. The maximum concentration of PAHs of $677.3 \mathrm{ng} / \mathrm{l}$ was recorded in the most contaminated site (station 6; AQ). This study indicated that the origin of contamination was pyrogenic in all sectors except Max was petrogenic. The present study declared that HMW PAHs are correlated significantly with most of the physicochemical parameters; while no correlations were found between LMW PAHs and most physicochemical parameters at low concentrations of PAHs.

\section{REFERENCES}

[1] C. D. Simpson, A. A. Mosi, W. R. Cullen and K. J. Reimer, "Composition and Distribution of Polycyclic Aromatic Hydrocarbons in Surficial Marine Sediments from Kitimat Harbour, Canada," The Science of the Total Environment, Vol. 181, No. 3, 1996, pp. 265-278.

[2] C. Seruto, Y. Sapozhnikova and D. Schlenk, "Evaluation of the Relationships between Biochemical Endpoints of PAH Exposure and Physiological Endpoints of Reproduction in Male California Halibut (Paralichthyscalifornicus) Exposed to Sediments from a Natural Oil Seep," Marine Environmental Research, Vol. 60, No. 4, 2005, pp. 454-465. http://dx.doi.org/10.1016/j.marenvres.2005.01.004

[3] Y. Qiu, G. Zhang, G. Liu, L. Guo, X. Li and O. Wai, "Polycyclic Aromatic Hydrocarbons (PAHs) in the Water Column and Sediment Core of Deep Bay, South China," Estuarine, Coastal and Shelf Science, Vol. 83, No. 1, 2009, pp. 60-66.

http://dx.doi.org/10.1016/j.ecss.2009.03.018

[4] R. Zoest and G. T. M. Van Eck, "Behaviour of Particulate Polychlorinated Biphenyls and Polycyclic Aromatic Hydrocarbons in the Scheldt Estuary, Netherlands," Sea Research, Vol. 26, No. 89-96, 1990, pp. 89-96.

[5] J. L. Zhou, T. W. Fileman, S. Evans, P. Donkin, R. F. C. Mantoura and S. J. Rowland, "Seasonal Distribution of Dissolved Pesticides and Polynuclear Aromatic Hydrocarbons in the Humber Estuary and Humber Coastal Zone," Marine Pollution Bulletin, Vol. 32, No. 8-9, 1996, pp. 599-608.

http://dx.doi.org/10.1016/0025-326X(96)00014-8

[6] J. L. Zhou and S. J. Rowland, "Evaluation of the Interactions between Hydrophobic Organic Pollutants and Suspended Particles in Estuarine Waters," Water Research, Vol. 31, No. 7, 1997, pp. 1708-1718.

http://dx.doi.org/10.1016/S0043-1354(96)00323-5
[7] M. B. Fernandes, M. A. Sicre, A. Boireau and J. Tronszynski, "Polyaromatic Hydrocarbon (PAH) Distributions in the Seine River and Its Estuary," Marine Pollution Bulletin, Vol. 34, No. 11, 1997, pp. 857-867. http://dx.doi.org/10.1016/S0025-326X(97)00063-5

[8] European Commission, "Directive of the European Parliament and of the Council 2000/60/EC Establishing a Framework for Community Action in the Field of Water Policy, Off," European Commission C513, 2000.

[9] Z. Zhanga, J. Huanga, G. Yua and H. Hong, “Occurrence of PAHs, PCBs and Organochlorine Pesticides in the Tonghui River of Beijing, China," Environmental Pollution, Vol. 130, No. 2, 2004, pp. 249-261. http://dx.doi.org/10.1016/j.envpol.2003.12.002

[10] M. I. Venkatesan and I. R. Kaplan, "Distribution and Transport of Hydrocarbons in Surface Sediments of the Alaskan Outer Continental Shelf," Geochimicaet Cosmochimica Acta, Vol. 46, No. 11, 1982, pp. 2135-2149. http://dx.doi.org/10.1016/0016-7037(82)90190-9

[11] M. J. Nieva-Cano, S. Rubio-Barroso and M. J. SantosDelgado, "Carcinogenic and Mutagenic Properties of PAHs," Analyst, Vol. 126, No. 8, 2001, pp. 132-140. http://dx.doi.org/10.1039/b102546p

[12] L. Tang, X. Y. Tang, Y. G. Zhu, M. H. Zheng and Q. L. Miao, "Contamination of Polycyclic Aromatic Hydrocarbons (PAHs) in Urban Soils in Beijing, China," Environment International, Vol. 31, No. 6, 2005, pp. 822-828. http://dx.doi.org/10.1016/j.envint.2005.05.031

[13] T. O. Said and M. A. F. Hamed, "Determination of Persistant Organic Pollutants in Water of New Damietta Harbour, Egypt," Egyptian Journal of Aquatic Research, Vol. 32, No. 1, 2006, pp. 235-245.

[14] W. Guo, M. He, Z. Yang, C. Lin, X. Quan and H. Wang, "Distribution of Polycyclic Aromatic Hydrocarbons in Water, Suspended Particulate Matter and Sediment from Daliao River Watershed, China," Chemosphere, Vol. 68, No. 1, 2007, pp. 93-104.

http://dx.doi.org/10.1016/j.chemosphere.2006.12.072

[15] J. M. Neff, "Polycyclic Aromatic Hydrocarbons in the Aquatic Environment: Sources, Fates and Biological Effects," Applied Science Publishers, London, 1979.

[16] I. Tolosa, S. Mora, M. R. Sheikholeslami, J. P. Villeneuve, J. Bartocci and C. Cattini, "Aliphatic and Aromatic Hydrocarbons in Coastal Caspian Sea Sediments," Marine Pollution Bulletin, Vol. 48, No. 1-2, 2004, pp. 44-60. http://dx.doi.org/10.1016/S0025-326X(03)00255-8

[17] N. Cardellicchio, A. Buccolieri, S. Giandomenico, L. Lopez, F. Pizzulli and L. Spada, "Organic Pollutants (PAHs, PCBs) in Sediments from the Mar Piccolo in Taranto, Ionian Sea, Southern Italy," Marine Pollution Bulletin, Vol. 55, No. 10-12, 2007, pp. 451-458. http://dx.doi.org/10.1016/j.marpolbul.2007.09.007

[18] M. K. Z. El Deeb, T. O. Said, M. H. El Naggar and M. A. Shreadah, "Distribution and Sources of Apliphatic and Polycyclic Aromatic Hydrocarbons in Surface Sediments, Fish and Bivalves of Abu Qir Bay," Bulletin of Environmental Contamination and Toxicology, Vol. 78, No. 5, 2007, pp. 373-379.

[19] M. K. Z. El Deeb, T. O. Said, M. H. El Naggar and M. A. 
Shreadah, "An Assessment of Pollution Status of Abu Qir Bay with Polycycic Aromatic Hydrocarbons (PAHs) and Their Possible Origins," Proceedings of the 8th International Conference on the Mediterranean Coastal Environment. MEDCOAST 2007, Alexandria, 13-17 November 2007, pp. 895-906.

[20] H. I. Emara, T. O. Said, N. A. El Naggar and. A. Shreadah, "Aliphatic and Polycyclic Hydrocarbon Compounds as Chemical Markers for Pollution Sources in Relation to Physicochemical Characteristics of the Eastern Harbour (Egyptian Mediterranean Sea)," Egyptian Journal of Aquatic Research, Vol. 34, No. 3, 2008, pp. 1-19.

[21] M. A. Shreadah, T. O. Said, A. M. Younis and R. S. Farag, "Speciation of Organotin Compounds in Sediments of Semi-Closed Areas along the Mediterranean Coast of Alexandria," Chemistry and Ecology, Vol. 22, No. 5, 2006, pp. 395-404.

[22] H. I. Emara and M. A. Shreadah, "Distribution and Composition of Aliphatic Aromatic Petroleum Hydrocarbons at Some Hot Spots of Alexandria Coastal Water, Egypt," International Workshop on Integrated Coastal Zone Management, Izmir, 20-22 October 2009, pp. 201-217.

[23] M. A. Shreadah, T. O. Said, M. I. Abdel Moniem, E. M. I. Fathallah and M. E. Mahmoud, "PAHs in Sediments along the Semi-Closed Areas of Alexandria, Egypt," Journal of Environmental Protection, Vol. 2, No. 6, 2011, pp. 700709.

[24] M. A. Shreadah, T. O. Said, I. M. Othman, E. M. I. Fathallah and M. E. Mahmoud, "Polychlorinated Biphenyls and Chlorinated Pesticides in Sediments along the SemiClosed Areas of Alexandria, Egypt," Journal of Environmental Protection (JEP), Vol. 3, No. 2, 2012, pp. 141149.

[25] T. O. Said and M. A. Hamed, "Mobility of Polycyclic Aromatic Hydrocarbons in Water of the Egyptian Red Sea Coasts," Bulletin Environmental Contaminant and Toxicology," Vol. 77, No. 1, 2006, pp. 126-136. http://dx.doi.org/10.1007/s00128-006-1041-8

[26] A. El Nemr and A. M. A. Abd-Allah, "Contamination of Polycyclic Aromatic Hydrocarbons (PAHs) in Microlayer and Subsurface Waters along Alexandria Coast, Egypt," Chemosphere, Vol. 52, No. 10, 2003 pp. 1711-1716. http://dx.doi.org/10.1016/S0045-6535(03)00300-X

[27] M. K. Z. EL-Deeb and H. I. Emara, "Polycyclic Aromatic Hydrocarbon and Aromatic Plasticizer material in the Sea Water of Alexandria Coastal Area, Egypt," Egyptian Journal of Aquatic Research, Vol. 31, 2005, pp. 15-24.

[28] S. D. Wahby and M. A. Shreadah, "The Effect of Sewage Discharge on Some Chemical Characteristics of Seawater. VII Journees Etud Pollutions, Lvcerene," CIESM, 1984, pp. 81-90.

[29] M. A. Shreadah and H. I. Emara, "The Distribution of Chromium, Copper, Cadmium, and Lead in Areas of Multi-Polluting Factors of Alexandria," International Proceedings of the Symposium on Market Chemistry in the Arab Region, Suez, April 1991, pp. 30-50.

[30] M. A. Shreadah and F. R. Tayel, "Environmental Condition of the Western Harbor of Alexandria: Physico-Che- mical Characteristics," Bulletin HIPH-High Institute of Public Health, Vol. XXII, No. 1, 1991, pp. 213-228.

[31] H. I. Emara, M. A. Shreadah, T. H. Maoustafa and M. S. El-Deek, "Effects of Sewage and Industrial Wastes on the Chemical Characterstics of the Eastern Harbor and ElMax Bay Waters of Alexandria, Egypt," Science of the Total Environment, Vol. 112, No. 2-3, 1992, pp. 773-784.

[32] M. A. Said, M. S. El-Deek, T. H. Mamoud and M. A. Shreadah, "Effect of Pollution on the Hydrochemical Characteristics of Different Water Types in El-Mex Bay Area West of Alexandria, Egypt," Acta Adriatica, Vol. 34, No. 1/2, 1994, pp. 9-19.

[33] F. R. Tayel and M. A. Shreadah, "Fe, Cu, Mn, Pb and Cd in Some Fish Species from the Western Harbor of Alexandria, Egypt," Bulletin of the National Institute of Oceanography and Fisheries, A. R. E., Vol. 22, 1996, pp. 8596.

[34] M. A. Shreadah, T. O. Said, A. M. Younis, and R. S. Farag, "Physico-Chemical Characteristics of the SemiClosed Areas along the Mediterranean Coast of Alexandria, Egypt," The Egyptian Journal of Aquatic Research, Vol. 32, 2006, pp. 38-48.

[35] A. H. Shobier, S. A. Abdel Ghani and M. A. Shreadah, "Distribution of Total Mercury in Sediments of Four Semi-Enclosed Basins along the Mediterranean Coast of Alexandria," The Egyptian Journal of Aquatic Research, Vol. 37, No. 1, 2011, pp. 1-11.

[36] M. A. Shredah, S. A. Abdel Ghani, A. Abdel Samie T., A. M. M. Ahmed and H. B. I. Hawash, "Mercury and Methyl Mercury in Sediments of Northern Lakes-Egypt," Journal of Environmental Protection, Vol. 3, No. 3, 2012, pp. 254-261. http://dx.doi.org/10.4236/jep.2012.33032

[37] M. S. Masoud, T. O. Said, G. El-Zokm and M. A. Shreadah, "Assesment of Heavy Metals Contamination in Surface Sediments of the Egyptian Red Sea Coasts," Asturalian Journal of Basic and Applied Sciences, Vol. 6, No. 6, 2012, pp. 44-58.

[38] S. Abdel Salam, G. El Zokm, A. Shobier, T. Othman, M. A. Shreadah, "Metal Pollution in Surface Sediments of Abu-Qir Bay and the Eastern Harbour of Alexandria, Egypt," The Egyptian Journal of Aquatic Research, Vol. 39, No. 1, 2013, pp. 1-12. http://dx.doi.org/10.1016/j.ejar.2013.03.001

[39] J. D. H. Strickland and T. R. Parsons, "A Practical Hand Book of Seawater Analysis," Bulletin of the Fisheries Research Board of Canada, Ottawa, 1975.

[40] FAO, "Manual of Methods in Aquatic Environmental Research, Part I: Permanganate Value (Oxidiability) of Organic Matter in Natural Waters," FAO Fisheries Technical paper, Vol. 137, 1976, pp. 169-174.

[41] T. R. Parsons, Y. Matia and G. M. Malli, "Determination of Petroleum Hydrocarbons, A Manual of Chemical and Biological Method for Seawater Analysis," Pergamon Press, Oxford, 1985.

[42] H. Sundberg, U. Tjarnlund, G. Akerman, M. Blomberg, R. Ishaq, K. Grunder, T. Hammar, D. Broman and L. Balk, "The Distribution and Relative Toxic Potential of Organic Chemicals in a PCB Contaminated Bay," Marine Pollu- 
tion Bulletin, Vol. 50, No. 2, 2005, pp. 195-207. http://dx.doi.org/10.1016/j.marpolbul.2004.10.010

[43] K. Maskaoui, J. L. Zhou, H. S. Hong and Z. L. Zhang, "Contamination by Polycyclic Aromatic Hydrocarbons in the Jiulong River Estuary and Western Xiamen Sea, China," Environmental Pollution, Vol. 118, No. 1, 2002, pp. 109-122. http://dx.doi.org/10.1016/S0269-7491(01)00208-1

[44] I. N. Nasr, M. H. Arief, A. H. Abdel-Aleem and F. M. Malhat, "Polycyclic Aromatic Hydrocarbons (PAHs) in Aquatic Environment at El-Menofiya Governorate, Egypt," Applied Sciences Research, Vol. 6, No. 1, 2010, pp. 1321.

[45] O. Duke, "Source Determination of Polynuclear Aromatic Hydrocarbons in Water and Sediment of a Creek in the
Niger Delta Region," African Journal of Biotechnology, Vol. 7, No. 3, 2008, pp. 282-285.

[46] A. H. Arias, C. V. Spetter, R. H. Freije and J. E. Marcovecchio, "Polycyclic Aromatic Hydrocarbons in Water, Mussels (Brachidontes sp., Tagelus sp.) and Fish (Odontesthes sp.) from Bahía Blanca Estuary, Argentina," Estuarine, Coastal and Shelf Science, Vol. 85, No. 1, 2009, pp. 67-81. http://dx.doi.org/10.1016/j.ecss.2009.06.008

[47] A. M. Stortini, T. Martellini, M. Del Bubba, L. Lepri, G. Capodaglio and A. Cincinelli, "n-Alkanes, PAHs and Surfactants in the Sea Surface Microlayer and Sea Water Samples of the Gerlache Inlet Sea, Antarctica," Microchemical Journal, Vol. 92, No. 1, 2009, pp. 37-43. http://dx.doi.org/10.1016/j.microc.2008.11.005 